\title{
Topical Interleukin 1 Receptor Antagonist for Treatment of Dry Eye Disease
}

\section{Citation}

Amparo, Francisco, Mohammad H. Dastjerdi, Andre Okanobo, Giulio Ferrari, Leila Smaga, Pedram Hamrah, Ula Jurkunas, Debra A. Schaumberg, and Reza Dana. 2013. "Topical Interleukin 1 Receptor Antagonist for Treatment of Dry Eye Disease." JAMA Ophthalmology 131 (6) (June 1): 715. doi:10.1001/jamaophthalmol.2013.195.

\section{Published Version}

10.1001/jamaophthalmol.2013.195

\section{Permanent link}

http://nrs.harvard.edu/urn-3:HUL.InstRepos:34814007

\section{Terms of Use}

This article was downloaded from Harvard University's DASH repository, and is made available under the terms and conditions applicable to Other Posted Material, as set forth at http:// nrs.harvard.edu/urn-3:HUL.InstRepos:dash.current.terms-of-use\#LAA

\section{Share Your Story}

The Harvard community has made this article openly available.

Please share how this access benefits you. Submit a story.

\section{Accessibility}




\title{
Topical Interleukin 1 Receptor Antagonist for Treatment of Dry Eye Disease:
}

\author{
A Randomized Clinical Trial \\ Francisco Amparo, MD, MSc" ${ }^{\#}$, Mohammad H. Dastjerdi, MD\#, Andre Okanobo, MD, Giulio \\ Ferrari, MD, Leila Smaga, BSc, Pedram Hamrah, MD, Ula Jurkunas, MD, Debra A. \\ Schaumberg, ScD, OD, MPH, and Reza Dana, MD, MPH, MSc \\ Department of Ophthalmology, Massachusetts Eye and Ear Infirmary (Drs Amparo, Dastjerdi, \\ Okanobo, Ferrari, Hamrah, Jurkunas, and Dana and Ms Smaga), and Division of Preventive \\ Medicine, Brigham and Women's Hospital (Dr Schaumberg), Harvard Medical School, Boston \\ \# These authors contributed equally to this work.
}

\begin{abstract}
Importance-The immunopathogenic mechanisms of dry eye disease (DED), one of the most common ophthalmic conditions, is incompletely understood. Data from this prospective, doublemasked, randomized trial demonstrate that targeting interleukin 1 (IL-1) by topical application of an IL-1 antagonist is efficacious in significantly reducing DED-related patient symptoms and corneal epitheliopathy.

Objective-To evaluate the safety and efficacy of treatment with the topical IL-1 receptor antagonist anakinra (Kineret; Amgen Inc) in patients having DED associated with meibomian gland dysfunction.
\end{abstract}

Design and Setting—Prospective phase 1/2, randomized, double-masked, vehicle-controlled clinical trial.

Participants-Seventy-five patients with refractory DED.

(C)2013 American Medical Association. All rights reserved.

Correspondence: Reza Dana, MD, MPH, MSc, Department of Ophthalmology, Massachusetts Eye and Ear Infirmary, 243 Charles St, Boston, MA 02114 (reza_dana@meei.harvard.edu)..

Author Contributions: Dr Dana had full access to all the data in the study and takes responsibility for the integrity and accuracy of the data and the corresponding data analysis.

Conflict of Interest Disclosures: Drs Dastjerdi and Dana report being listed as coinventors in a patent application pertaining to use of IL-1 blockers for ophthalmic use, licensed by Schepens Eye Research Institute and Massachusetts Eye and Ear Infirmary to Eleven Biotherapeutics Inc. Dr Schaumberg reports receiving support from Pfizer Inc; holding investments in Mimetogen and TearLab; being a consultant to Alcon, Allergan, Celtic, Eleven Biotherapeutics Inc, Inspire, Mimetogen, Pfizer Inc, and SARcode Bioscience; holding a proprietary interest in patent 11873478 covering compositions and methods for treating eye disorders and conditions; and receiving honoraria from Allergan and Pfizer Inc. Dr Dana reports holding equity in Eleven Biotherapeutics Inc.

Online-Only Material: The eTables and eFigure are available at http://www.jamaophth.com.

Trial Registration

clinicaltrials.gov Identifier: NCT00681109 
Interventions-Participants were randomized to receive treatment with topical anakinra, 2.5\% $(n=30)$, anakinra, $5 \%(n=15)$, or vehicle $(1 \%$ carboxymethylcellulose $)(n=30) 3$ times daily for 12 weeks.

Main Outcomes and Measures-Primary outcomes were corneal fluorescein staining (CFS), complete bilateral CFS clearance, dry eye-related symptoms as measured by the Ocular Surface Disease Index, tear film breakup time, and meibomian gland secretion quality.

Results-Topical anakinra was well tolerated compared with vehicle, with no reports of serious adverse reactions attributable to the therapy. After 12 weeks of therapy, participants treated with anakinra, $2.5 \%$, achieved a $46 \%$ reduction in their mean CFS score ( $P=.12$ compared with vehicle and $P<.001$ compared with baseline); participants treated with anakinra, $5 \%$, achieved a $17 \%$ reduction in their mean CFS score $(P=.88$ compared with vehicle and $P=.33$ compared with baseline); and patients treated with vehicle achieved a $19 \%$ reduction in their mean CFS score $(P=.11)$. Complete bilateral CFS clearance was noted in 8 of 28 patients (29\%) treated with anakinra, $2.5 \%$, vs in 2 of 29 patients $(7 \%)$ treated with vehicle $(P=.03)$. By week 12 , treatment with anakinra, $2.5 \%$, and treatment with anakinra, $5 \%$, led to significant reductions in symptoms of $30 \%$ and $35 \%$, respectively ( $P=.02$ and $P=.01$, respectively, compared with vehicle); treatment with vehicle led to a $5 \%$ reduction in symptoms.

Conclusions and Relevance-Treatment with topical anakinra, 2.5\%, for 12 weeks was safe and significantly reduced symptoms and corneal epitheliopathy in patients with DED. These data suggest that the use of an IL-1 antagonist may have a role as a novel therapeutic option for patients with DED.

Dry eye disease (DED) is a multifactorial disorder of the tears and ocular surface that results in symptoms of discomfort, visual disturbance, and potentially damaging ocular surface inflammation. ${ }^{1,2}$ It is caused by deficient tear production, excessive tear evaporation, or both. Meibomian gland dysfunction is thought to be the leading cause of DED, and it can exist alone or concurrently with tear-deficient forms of DED. ${ }^{1,3}$ Meibomian glands are located in the eyelids and drain onto the ocular surface, where their oily secretion (meibum) prevents premature evaporation of the tear film.

An estimated 9 million persons in the United States alone are affected by DED; millions more may have a milder form of the disease or may experience symptoms of dry eye when exposed to predisposing conditions such as low humidity or contact lens use. ${ }^{1,3,4}$ The prevalence of DED among the middle-aged or older population is approximately $7.8 \%$ for women and $4.5 \%$ for men in the United States, ${ }^{3-5}$ and the results of some studies ${ }^{1,3}$ suggest that the prevalence may be considerably higher among the Asian population. Dry eye disease can adversely affect the quality of life by disabling performance of activities of daily living, such as reading and driving. ${ }^{5}$ Remarkably, as suggested by other study ${ }^{6}$ findings, DED and moderate an-gina produce comparable reductions in patient-reported quality of life, while severe DED can reduce the quality of life to a greater extent than a hip fracture. It is estimated that up to $29 \%$ of patients seen for outpatient ophthalmic examination may exhibit clinically relevant signs or symptoms of DED; therefore, DED is likely the most common reason for which patients seek ophthalmological care in the developed world. ${ }^{7}$ One 
study $^{8}$ estimated a mean of more than $\$ 55$ billion in annual direct and indirect costs of DED to society in the United States alone.

The precise pathogenic mechanisms of DED are incompletely understood; however, it is recognized that desiccating stress induces ocular surface inflammation that contributes significantly to the signs (epitheliopathy) and symptoms of DED. ${ }^{1}$ The proinflammatory cytokine interleukin 1 (IL-1) is intimately involved in the regulation of ocular surface inflammation, including in the setting of DED. ${ }^{9-13}$ Interleukin 1 promotes the activation and migration of leukocytes, the expansion of pathogenic $\mathrm{T}$ cells, and the production of other proinflammatory cytokines that mediate disease. ${ }^{14,15}$ Anti-inflammatory medications, such as tetracycline derivatives, topical corticosteroids, and cyclosporine $\mathrm{A}$, that have been successfully used in the treatment of DED downregulate the production of IL-1 and upregulate the production of IL-1 receptor antagonist (IL-1Ra) at the ocular surface. ${ }^{13,16}$ The IL-1Ra suppresses IL-1-mediated inflammation by competitively inhibiting the binding of IL-1 $\alpha$ and IL-1 $\beta$ to IL-1 receptor I. ${ }^{17}$ Topical IL-1Ra has been successfully used to treat experimental rodent models of corneal transplant rejection, allergic conjunctivitis, and alkali burn- associated ocular inflammation. ${ }^{18-20}$

Anakinra (Kineret; Amgen Inc) is a recombinant version of human IL-1Ra approved for treatment of rheumatoid arthritis. ${ }^{17}$ In addition, anakinra has been used off label to treat many conditions that involve IL-1-mediated inflammation. ${ }^{21}$ A 2012 study 22 showedthattopicalanakinra was effective in treating DED in an animal model. Given that IL-1 has been implicated in the immunopathogenic mechanisms of DED-associated ocular surface inflammation, we hypothesized that the targeted inhibition of IL-1 may prove effective in treatment of DED associated with meibomian gland dysfunction, the most common type of DED. ${ }^{2}$ Herein, we present the results of a randomized, double-masked clinical trial designed to assess the safety and efficacy of topical IL-1Ra in patients with DED.

\section{METHODS}

\section{STUDY DESIGN AND PARTICIPANTS}

This was a prospective phase $1 / 2,16$-week, randomized, double-masked clinical trial to evaluate the safety and efficacy of a topical application of anakinra in patients with meibomian gland dysfunction-associated DED. Seventy-five participants were randomly allocated in a 2:2:1 ratio to receive vehicle, anakinra, $2.5 \%$, or anakinra, $5 \%$, using a permuted-block design. Permuted blocks were obtained using a computer-based random code generator (Research Randomizer; http://randomizer.org/). Randomization was performed by the Massachusetts Eye and Ear Infirmary's pharmacy, and neither participants nor research staff were aware of the assigned treatments.

Eligible participants were 18 years or older having symptoms of DED with positive corneal or conjunctival epithelial staining (score, $\geq 1$ ) in either eye on the modified Oxford Grading Scale; this grading system is based on a 6-category diagram (score range, 0-5, representing increasing disease severity) that is compared with the eye's staining pattern. ${ }^{23}$ Additional inclusion criteria were a diagnosis of meibomian gland dysfunction with or without aqueous 
tear deficiency, ${ }^{24}$ normal eyelid position and closure, and a negative urine pregnancy test result in conjunction with the use of contraceptives for women of childbearing age. Exclusion criteria were the following: any history of eyelid surgery, a history of treatment with anakinra, ocular surgery within the past 3 months, active ocular infection or ocular allergies, a history of cicatrizing ocular surface disease, the use of isotretinoin within the previous 6 months, corneal epithelial defect larger than $1 \mathrm{~mm}^{2}$ in either eye, active or recently ( $<3$ months) diagnosed liver or renal disease, the use of topical cyclosporine or corticosteroids within 2 weeks of enrollment, and changes in dosage of oral tetracycline compounds within 1 month of enrollment. Except for a 2-week wash-out period of topical corticosteroids and topical cyclosporine before enrollment, participants were permitted to continue their chronic treatments, including the use of artificial tears, eyelid massage, or warm compresses.

Study approval was obtained from the Human Studies Committee of the Massachusetts Eye and Ear Infirmary, as well as an Investigational New Drug approval (IND 101 168) from the US Food and Drug Administration. Informed consent was obtained from all patients after the nature and possible consequences of treatment were explained. Research was conducted in accord with the requirements of the Health Insurance Portability and Accountability Act and the tenets of the Declaration of Helsinki.

\section{OUTCOME MEASURES}

The primary end points after 12 weeks of treatment included the following: a mean reduction in corneal epitheliopathy as measured by corneal fluorescein staining (CFS), the proportion of patients achieving complete bilateral CFS clearance after treatment (salinemoistened 1\% fluorescein strips [FUL-GLO; Akorn, Inc] were used to instill fluorescein in the inferior palpebral conjunctiva; scoring was performed after 3 minutes using slitlamp and cobalt blue illumination), a change in tear film breakup time, changes in meibomian gland secretion quality, ${ }^{24}$ and a mean reduction in DED symptoms as measured by the Ocular Surface Disease Index (OSDI). ${ }^{25}$ Nonprimary end points included visual acuity, the number of expressible meibomian glands among 10 examined, and tear secretion as measured by the Schirmer test using anesthesia. Safety end points included ocular and systemic adverse events related to the use of the study drug. A random subset of participants (10 from the vehicle arm and 17 from the anakinra arms) underwent conjunctival and eyelid margin cultures for microbiologic workup at baseline and at week 12 to assess potential changes in bacterial flora.

\section{STUDY TREATMENT}

Participants were randomly assigned in a 2:1:2 ratio to receive anakinra, $2.5 \%$, anakinra, $5 \%$, or vehicle (1\% carboxymethyl-cellulose [Refresh Liquigel; Allergan Inc]). For the active treatment, anakinra protein was solubilized in a formulation compound identical to the one used as treatment for the vehicle arm. We instructed patients to instill 1 drop 3 times daily for 12 weeks in both eyes. Anakinra, 2.5\%, and anakinra, 5\%, ophthalmic solutions were prepared at the Massachusetts Eye and Ear Infirmary's pharmacy under the US Pharmacopeia standards for pharmaceutical compounding, and all vials were sampled for microbiologic testing. 
Clinical visits were scheduled at days 0 and 1 and at weeks 2, 6, 12, and 16. A safety visit at day 1 included a complete eyelid, ocular surface, and anterior segment examination to assess for signs and symptoms of toxic effects related to the use of the study drug. Subsequent visits included completion of the OSDI questionnaire and a complete eye examination. Treatment was discontinued after 12 weeks, and a final evaluation was performed at week 16.

\section{STATISTICAL ANALYSIS}

We assessed treatment efficacy based on the intent-to-treat principle using each participant's randomized treatment assignment to define treatment groups and outcomes. Safety variables were analyzed with regard to treatment that participants actually received (the safety population). We calculated patient scores for all outcome variables by averaging the data from both eyes, except for the OSDI questionnaire (in which a single score was obtained per participant) and complete bilateral CFS clearance (defined by the absence of CFS in both eyes). The OSDI questionnaire comprises the following 3 subscales: bothersome symptoms, visual function, and environmental triggers. The derived index of 0 to 100 is calculated from the total number of questions answered. ${ }^{25}$ Four participants treated with anakinra and 2 participants treated with vehicle had visually significant cataracts. In addition, 1 participant with diabetes mellitus in the anakinra, 5\%, arm who developed uncontrolled hyperglycemia during the study reported daily visual fluctuations. For these 7 participants, the visual function- related questions were excluded, and the OSDIs were adjusted accordingly. We defined outcome measures on the basis of the change for each participant from baseline to each follow-up point, controlling for chance imbalances in the mean baseline values between treatment groups. The last-observation-carried-forward method was used in the few cases of missing data (eg, participant withdrawal and missed visit). To assess the effect of treatment, we compared the distribution of scores for each outcome measure between each anakinra dose group vs the vehicle group using the $t$ test for continuous and ordinal variables and the $\chi^{2}$ test for categorical variables. Two-sided $P<.05$ was considered statistically significant for all comparisons.

\section{RESULTS}

Seventy-five patients were enrolled and randomly assigned to 1 of 3 treatment arms in a double-masked fashion (Figure 1). Baseline demographic and ocular characteristics between study groups were balanced (Table 1).

\section{CORNEAL FLUORESCEIN STAINING}

By week 6, CFS was reduced by $30 \%$ in the anakinra, $2.5 \%$, group $(P=.01$ compared with baseline and $P=.33$ compared with vehicle); by $29 \%$ in the anakinra, 5\%, group ( $P=.10$ compared with baseline and $P=.38$ compared with vehicle); and by $15 \%$ in the vehicle group $(P=.10)$. Respective reductions in the mean CFS scores after 12 weeks of therapy were $46 \%$ (95\% CI, -1.1 to -0.4$)$ in the anakinra, $2.5 \%$, group $(P=.12$ compared with vehicle and $P<.001$ compared with baseline); $17 \%$ ( -0.7 to 0.2$)$ in the anakinra, $5 \%$, group $(P=.88$ compared with vehicle and $P=.33$ compared with baseline); and $19 \%$ (- 
0.70 to 0.04$)$ in the vehicle group $(P=.11)$ (Table 2, Figure 2A, and eFigure 1 [http:// www.jamaophth.com]).

By week 12, complete bilateral CFS clearance (compared with baseline) was achieved among 8 of 28 patients (29\%) in the anakinra, 2.5\%, group; among 1 of $15(7 \%)$ in the anakinra, 5\%, group; and among 2 of $29(7 \%)$ in the vehicle group $(P=.03$ and $P=.71$, respectively, compared with vehicle) (Table 2 and Figure 2B). After 12 weeks, patients in the anakinra, $2.5 \%$, group were 3 times more likely to achieve complete bilateral CFS clearance than were patients in the vehicle group (relative risk difference, $3.3 ; 95 \% \mathrm{CI}$, 1.0-10.9; absolute risk difference, 0.23 ; 0.03-0.40).

On termination of anakinra application at week 12, a clear regression occurred, especially in participants receiving anakinra, $2.5 \%$, toward baseline values for CFS and for complete bilateral CFS clearance. These results are shown in Figure 2A and B and in eFigure 1.

\section{PATIENT SYMPTOMS}

Symptom reduction in response to anakinra treatment was evident as early as week 2 and reached statistical significance ( $P=.02$ for both anakinra, $2.5 \%$, and anakinra, $5 \%)$ compared with vehicle by week 6 . By week 12 , dry eye symptoms were reduced by $30 \%$ (95\% CI, -21.1 to -6.4$)$ in the anakinra, $2.5 \%$, group $(P=.02$ compared with vehicle) and by $35 \%(-27.9$ to -5.7$)$ in the anakinra, $5 \%$, group $(P=.01)$; symptoms in the vehicle group were reduced by 5\% (-8.4 to 3.7) (Table 2 and Figure 3A). The use of anakinra, $2.5 \%$, led to statistically significant decreases vs vehicle in all 3 subscales of the OSDI, and the use of anakinra, $5 \%$, led to statistically significant decreases vs vehicle in 2 subscales of the OSDI (Figure 3B-D).

\section{OTHER PRIMARY END POINTS}

By week 12, tear film breakup time increased (improved) by $18 \%$ (95\% CI, -0.4 to 1.7 ) in the anakinra, $2.5 \%$, group; by $11 \%$ (-1.0 to 1.8$)$ in the anakinra, $5 \%$, group; and by $16 \%$ ( -0.2 to 1.5$)$ in the vehicle group. However, no significant differences were observed for any group vs baseline or for either treatment arm vs vehicle (Table 2). Meibomian gland secretion quality tended to improve during 12 weeks compared with baseline by $11 \%$ (95\% $\mathrm{CI},-0.5$ to 0.1$)$ in the anakinra, $2.5 \%$, group ( $P=.27$ vs baseline and $P=.89$ vs vehicle); by $5 \%$ ( -0.5 to 0.3$)$ in the anakinra, $5 \%$, group $(P=.53$ vs baseline and $P=.68$ vs vehicle); and by $11 \%(-0.40$ to -0.03$)$ in the vehicle group $(P=.02$ vs baseline $)$.

\section{NONPRIMARY END POINTS}

The mean Schirmer test scores did not substantively change in any group $(P=.45$ and $P=$. 24 , respectively, for anakinra, $2.5 \%$, and anakinra, $5 \%$, vs vehicle) (Table 2). The number of expressible meibomian glands among 10 examined increased slightly (6\%) only in the anakinra, $2.5 \%$, group ( $P=.09$ compared with vehicle). During the 12-week study, the mean visual acuity improved in participants treated with anakinra, $2.5 \%$, and with anakinra, $5 \%(P$ $=.05$ and $P=.16$, respectively, compared with vehicle) and decreased in participants treated with vehicle. Intraocular pressure did not change significantly in any of the treatment arms. After 12 weeks, a statistically significant reduction was observed in the frequency of 
artificial tear use by the anakinra, $2.5 \%$, group $(P<.01$ vs baseline $)$ compared with the vehicle group $(P=.29)$ and the anakinra, 5\%, group $(P=.34)$.

\section{ADVERSE EVENTS}

No serious ocular or systemic adverse events attributable to treatment occurred during the study (eTable 1). Eight participants (6 in the vehicle group and 2 in the anakinra, 2.5\%, group) failed to complete 16 weeks of the study. In the vehicle group, 3 patients terminated application of the eyedrops because of their perception of lack of improvement. A fourth patient underwent cataract surgery and opted to terminate treatment at week 8 . Two participants completed the 12-week treatment period but did not return for the week 16 visit. In the anakinra, $2.5 \%$, group, 2 participants failed to complete the study visits. The first participant withdrew at week 3 after developing adenoviral conjunctivitis (at week 2). A second participant completed all treatment visits through week 12 but died of complications from chronic myelocytic leukemia before the week 16 visit. Overall, 3 participants were diagnosed as having conjunctivitis based on clinical examination, 1 in each treatment arm. Of these, 2 were diagnosed as having adenoviral conjunctivitis (one in the vehicle group at week 6 and another in the anakinra, 2.5\%, group at week 2). At week 11, a local ophthalmologist diagnosed conjunctivitis in a third participant (in the anakinra, 5\%, group). All cases resolved quickly, and culture results were negative.

\section{DRUG TOLERABILITY}

In addition to assessing symptom responses to therapy using the OSDI questionnaire, participants were asked about sensations of discomfort related to study drop instillation. Reports of all types of discomfort related to instillation of the study drops were more common in the vehicle group (eTable 1).

\section{MICROBIOLOGIC STUDIES}

In microbiologic studies performed on a random subset of patients in the 3 arms of the study at baseline and at week 12, eyelid margin culture positivity was higher than conjunctival culture positivity at baseline and at week 12 (eTable 2). No statistically significant differences in bacterial growth rates were observed among the 3 study arms.

\section{COMMENT}

Dry eye disease is a chronic inflammatory condition of the ocular surface induced by desiccating stress. ${ }^{1}$ Clinical and experimental animal model data indicate that DED is associated with significant overexpression of inflammatory cytokines, including IL-1, in the tear film, exocrine tissues, and surface epithelia. ${ }^{26}$ However, the options to treat the inflammatory component of DED are limited. The adverse effect profiles of several of the anti-inflammatory treatments used include ocular hypertension and cataracts from corticosteroid use, corneal melts from the use of nonsteroidal anti-inflammatory drugs, and persistent burning among some patients using topical cyclosporine. ${ }^{16,23,27}$

There is strong biologic rationale for targeting IL-1 in DED. ${ }^{12,28,29}$ Critical immunopathogenic mechanisms of DED include activation and mobilization of ocular 
surface antigen-presenting cells, as well as expansion and homing of effector $\mathrm{T}$ cells to the ocular surface ${ }^{30-33}$; these are mechanisms for which IL-1 has a critical role. ${ }^{17,34}$ Levels of proinflammatory forms of IL- 1 are directly correlated with the intensity of CFS, ${ }^{12}$ and IL-1 $\beta$ contributes to the loss of corneal epithelial barrier function associated with ocular surface inflammation. ${ }^{35}$ Most important, in the context of DED, IL-1 is a principal mediator of nociception in peripheral tissues. ${ }^{36}$

In this 12-week, randomized, double-masked trial, anakinra, 2.5\%, was well tolerated and was significantly more effective than vehicle in improving the signs (corneal clearance of staining) and symptoms of DED. Anakinra, 2.5\%, was 4 times more likely $(P=.03)$ than vehicle to induce complete bilateral CFS clearance, a result that (to our knowledge) has not been reported in any other dry eye treatment trial to date and which underscores the protective effect of IL-1 antagonism for the epithelium in DED.

Topical anakinra also significantly reduced dry eye symptoms 6 times more effectively than the vehicle control, which is a lubricant capable of independently improving the signs and symptoms of DED, ${ }^{37}$ as measured by the OSDI, a questionnaire widely used in clinical trials to measure patient-reported symptoms of DED. Moreover, termination of anakinra (but not vehicle) application at week 12 led to a clear trend toward increased symptoms between weeks 12 and 16, confirming the therapeutic effect of the drug.

We cannot elucidate precisely the reasons behind the apparent regression of effect for the higher (5\%) dose of anakinra in treating corneal epitheliopathy at the single time point of week 12, despite significant effects at weeks 2 and 6 and the progressive and significant effect in symptom reduction at weeks 2,6 , and 12 . Two issues deserve mention in this regard. First, there is good biological rationale for speculating that IL-1Ra may, at least in part, address epithelial disease (as measured by staining) independent of the perception of discomfort given the critical role of IL-1 in mediating pain. ${ }^{36}$ This may explain the rapid response in symptom reduction seen with anakinra at both concentrations. Indeed, the fact that the symptom response curves to these concentrations are remarkably similar suggests near-maximal receptor occupancy with both concentrations, likely implying considerable room for lowering the concentration of IL-1Ra without appreciable loss in efficacy. Second, the formulation of anakinra used in this trial was not optimized for stability at the high protein concentrations used. At high concentrations, maintaining the solubility of anakinra requires anionic excipients to prevent aggregation and precipitation, which were not used in this study. ${ }^{38}$ For these reasons and given the few patients $(n=15)$ treated with the higher concentration, we are unable to make any strong conclusions regarding a dose-response effect.

Several end points, including the Schirmer test scores, did not change significantly as a result of anakinra therapy despite the responses noted in relation to surface epitheliopathy and symptoms. This suggests that IL-1 blockade, at least during the 12-week period tested herein, has a direct therapeutic effect on the ocular surface rather than on the tear film or tear-producing glands. This conclusion is in accord with other studies ${ }^{23,27,39}$ that reported the lack of a strong correlation between tear-based and ocular surface end points following treatment for DED. In addition, anakinra use did not induce any significant changes in 
intraocular pressure, a key issue given the strong risk of pressure elevation and the risk of glaucoma with corticosteroid use. ${ }^{16}$

Visual disturbance can occur in DED secondary to abnormal tear film composition and the loss of ocular surface integrity. 1,5,6,40,41 This visual disturbance is typically associated with prolonged or demanding visual tasks, such as driving or computer use, rather than a significant drop in visual acuity as tested using a high-contrast chart in a dimly lit room. ${ }^{23,40}$ Notably, IL-1 blockade in our study was associated with a subtle but detectable improvement in visual acuity $(P=.05)$ using a high-contrast chart.

Interleukin 1 blockade did not increase the rate of ocular surface infections or bacterial growth compared with vehicle. Arguably, the epithelial recovery induced by IL-1 blockade may contribute to the prevention of bacterial adhesion to the ocular surface and resultant infection. ${ }^{42}$ Nonspecific symptoms of discomfort following eyedrop instillation are characteristic of patients with DED and are presumed to be due to loss of the epithelial barrier function and stimulation of nerve endings in the cornea ${ }^{43}$ The positive tolerability data associated with anakinra drop application and the associated reduction in symptoms over time suggest that IL-1 blockade can significantly attenuate the perception of ocular surface pain.

In conclusion, the results of this study provide proof-of-concept support for the therapeutic efficacy of topical IL-1 blockade in ameliorating the signs and symptoms of DED. To our knowledge, the topical use of a protein-based biologic agent in treatment of DED is unprecedented. The promising results from this study may herald a new era of highly targeted topical molecular treatments in treating ocular surface diseases.

\section{Acknowledgments}

Funding/Support: This study was supported in part by grant K24 EY019098 from the National Institutes of Health (Drs Amparo, Dastjerdi, Okanobo, Ferrari, and Dana and Ms Smaga).

\section{REFERENCES}

1. International Dry Eye WorkShop Study Group. The definition and classification of dry eye disease: report of the Definition and Classification Subcommittee of the International Dry Eye WorkShop (2007). Ocul Surf. 2007; 5(2):75-92. [PubMed: 17508116]

2. Nichols KK, Foulks GN, Bron AJ, et al. The International Workshop on Meibomian Gland Dysfunction: executive summary. Invest Ophthalmol Vis Sci. 2011; 52(4):1922-1929. [PubMed: 21450913]

3. Schaumberg DA, Sullivan DA, Buring JE, Dana MR. Prevalence of dry eye syndrome among US women. Am J Ophthalmol. 2003; 136(2):318-326. [PubMed: 12888056]

4. Schaumberg DA, Dana R, Buring JE, Sullivan DA. Prevalence of dry eye disease among US men: estimates from the Physicians' Health Studies. Arch Ophthalmol. 2009; 127(6):763-768. [PubMed: 19506195]

5. Miljanović B, Dana R, Sullivan DA, Schaumberg DA. Impact of dry eye syndrome on vision-related quality of life. Am J Ophthalmol. 2007; 143(3):409-415. [PubMed: 17317388]

6. Schiffman RM, Walt JG, Jacobsen G, Doyle JJ, Lebovics G, Sumner W. Utility assessment among patients with dry eye disease. Ophthalmology. 2003; 110(7):1412-1419. [PubMed: 12867401] 
7. Gupta N, Prasad I, Jain R, D'Souza P. Estimating the prevalence of dry eye among Indian patients attending a tertiary ophthalmology clinic. Ann Trop Med Parasitol. 2010; 104(3):247-255. [PubMed: 20507698]

8. Yu J, Asche CV, Fairchild CJ. The economic burden of dry eye disease in the United States: a decision tree analysis. Cornea. 2011; 30(4):379-387. [PubMed: 21045640]

9. Fabre EJ, Bureau J, Pouliquen Y, Lorans G. Binding sites for human interleukin 1a, $\gamma$-interferon and tumor necrosis factor on cultured fibroblasts of normal cornea and keratoconus. Curr Eye Res. 1991; 10(7):585-592. [PubMed: 1914496]

10. Rosenbaum JT, Planck ST, Huang XN, Rich L, Ansel JC. Detection of mRNA for the cytokines, interleukin-1a and interleukin-8, in corneas from patients with pseudophakic bullous keratopathy. Invest Ophthalmol Vis Sci. 1995; 36(10):2151-2155. [PubMed: 7657553]

11. Pflugfelder SC, Jones D, Ji Z, Afonso A, Monroy D. Altered cytokine balance in the tear fluid and conjunctiva of patients with Sjögren's syndrome keratoconjunctivitis sicca. Curr Eye Res. 1999; 19(3):201-211. [PubMed: 10487957]

12. Solomon A, Dursun D, Liu Z, Xie Y, Macri A, Pflugfelder SC. Pro- and anti-inflammatory forms of interleukin-1 in the tear fluid and conjunctiva of patients with dry-eye disease. Invest Ophthalmol Vis Sci. 2001; 42(10):2283-2292. [PubMed: 11527941]

13. De Paiva CS, Corrales RM, Villarreal AL, et al. Corticosteroid and doxycycline suppress MMP-9 and inflammatory cytokine expression, MAPK activation in the corneal epithelium in experimental dry eye. Exp Eye Res. 2006; 83(3):526-535. [PubMed: 16643899]

14. Fini ME, Girard MT. Expression of collagenolytic/gelatinolytic metalloproteinases by normal cornea. Invest Ophthalmol Vis Sci. 1990; 31(9):1779-1788. [PubMed: 2170294]

15. Sha Y, Markovic-Plese S. A role of IL-1R1 signaling in the differentiation of Th17 cells and the development of autoimmune diseases. Self Nonself. 2011; 2(1):35-42. [PubMed: 21776333]

16. Solomon A, Rosenblatt M, Li DQ, et al. Doxycycline inhibition of interleukin-1 in the corneal epithelium. Invest Ophthalmol Vis Sci. 2000; 41(9):2544-2557. [PubMed: 10937565]

17. Dinarello CA. Interleukin-1 in the pathogenesis and treatment of inflammatory diseases. Blood. 2011; 117(14):3720-3732. [PubMed: 21304099]

18. Yamada J, Zhu SN, Streilein JW, Dana MR. Interleukin-1 receptor antagonist therapy and induction of anterior chamber-associated immune deviation-type tolerance after corneal transplantation. Invest Ophthalmol Vis Sci. 2000; 41(13):4203-4208. [PubMed: 11095616]

19. Keane-Myers AM, Miyazaki D, Liu G, Dekaris I, Ono S, Dana MR. Prevention of allergic eye disease by treatment with IL-1 receptor antagonist. Invest Ophthalmol Vis Sci. 1999; 40(12): 3041-3046. [PubMed: 10549671]

20. Yamada J, Dana MR, Sotozono C, Kinoshita S. Local suppression of IL-1 by receptor antagonist in the rat model of corneal alkali injury. Exp Eye Res. 2003; 76(2):161-167. [PubMed: 12565803]

21. Le Loët X, Nordström D, Rodriguez M, et al. Effect of anakinra on functional status in patients with active rheumatoid arthritis receiving concomitant therapy with traditional disease modifying antirheumatic drugs: evidence from the OMEGA Trial. J Rheumatol. 2008; 35(8):1538-1544. [PubMed: 18634163]

22. Okanobo A, Chauhan SK, Dastjerdi MH, Kodati S, Dana R. Efficacy of topical blockade of interleukin-1 in experimental dry eye disease. Am J Ophthalmol. 2012; 154(1):63-71. [PubMed: 22541929]

23. Sall K, Stevenson OD, Mundorf TK, Reis BL, CsA Phase 3 Study Group. Two multicenter, randomized studies of the efficacy and safety of cyclosporine ophthalmic emulsion in moderate to severe dry eye disease. Ophthalmology. 2000; 107(4):631-639. [published correction appears in Ophthalmology. 2000;107(7):1220]. [PubMed: 10768324]

24. Foulks GN, Bron AJ. Meibomian gland dysfunction: a clinical scheme for description, diagnosis, classification, and grading. Ocul Surf. 2003; 1(3):107-126. [PubMed: 17075643]

25. Schiffman RM, Christianson MD, Jacobsen G, Hirsch JD, Reis BL. Reliability and validity of the Ocular Surface Disease Index. Arch Ophthalmol. 2000; 118(5):615-621. [PubMed: 10815152]

26. Stevenson W, Chauhan SK, Dana R. Dry eye disease: an immune-mediated ocular surface disorder. Arch Ophthalmol. 2012; 130(1):90-100. [PubMed: 22232476] 
27. Perry HD, Solomon R, Donnenfeld ED, et al. Evaluation of topical cyclosporine for the treatment of dry eye disease. Arch Ophthalmol. 2008; 126(8):1046-1050. [PubMed: 18695097]

28. Chotikavanich S, de Paiva CS, Li DQ, et al. Production and activity of matrix metalloproteinase-9 on the ocular surface increase in dysfunctional tear syndrome. Invest Ophthalmol Vis Sci. 2009; 50(7):3203-3209. [PubMed: 19255163]

29. Goyal S, Chauhan SK, Zhang Q, Dana R. Amelioration of murine dry eye disease by topical antagonist to chemokine receptor 2. Arch Ophthalmol. 2009; 127(7):882-887. [PubMed: 19597109]

30. Li DQ, Luo L, Chen Z, Kim HS, Song XJ, Pflugfelder SC. JNK and ERK MAP kinases mediate induction of IL-1 $\beta$, TNF- $\alpha$ and IL-8 following hyperosmolar stress in human limbal epithelial cells. Exp Eye Res. 2006; 82(4):588-596. [PubMed: 16202406]

31. El Annan J, Chauhan SK, Ecoiffier T, Zhang Q, Saban DR, Dana R. Characterization of effector T cells in dry eye disease. Invest Ophthalmol Vis Sci. 2009; 50(8):3802-3807. [PubMed: 19339740]

32. De Paiva CS, Chotikavanich S, Pangelinan SB, et al. IL-17 disrupts corneal barrier following desiccating stress. Mucosal Immunol. 2009; 2(3):243-253. [PubMed: 19242409]

33. Zheng X, de Paiva CS, Li DQ, Farley WJ, Pflugfelder SC. Desiccating stress promotion of Th17 differentiation by ocular surface tissues through a dendritic cell-mediated pathway. Invest Ophthalmol Vis Sci. 2010; 51(6):3083-3091. [PubMed: 20130281]

34. Wilson SE, Esposito A. Focus on molecules: interleukin-1: a master regulator of the corneal response to injury. Exp Eye Res. 2009; 89(2):124-125. [PubMed: 19254714]

35. Kimura K, Teranishi S, Nishida T. Interleukin-1 $\beta$-induced disruption of barrier function in cultured human corneal epithelial cells. Invest Ophthalmol Vis Sci. 2009; 50(2):597-603. [PubMed: 19171646]

36. Gabay E, Wolf G, Shavit Y, Yirmiya R, Tal M. Chronic blockade of interleukin-1 (IL-1) prevents and attenuates neuropathic pain behavior and spontaneous ec-topic neuronal activity following nerve injury. Eur J Pain. 2011; 15(3):242-248. [PubMed: 20801063]

37. Grene RB, Lankston P, Mordaunt J, Harrold M, Gwon A, Jones R. Unpreserved carboxymethylcellulose artificial tears evaluated in patients with keratoconjunctivitis sicca. Cornea. 1992; 11(4):294-301. [PubMed: 1424648]

38. Raibekas AA, Bures EJ, Siska CC, Kohno T, Latypov RF, Kerwin BA. Anion binding and controlled aggregation of human interleukin-1 receptor antagonist. Biochemistry. 2005; 44(29): 9871-9879. [PubMed: 16026159]

39. Barber LD, Pflugfelder SC, Tauber J, Foulks GN. Phase III safety evaluation of cyclosporine $0.1 \%$ ophthalmic emulsion administered twice daily to dry eye disease patients for up to 3 years. Ophthalmology. 2005; 112(10):1790-1794. [PubMed: 16102833]

40. Goto E, Yagi Y, Matsumoto Y, Tsubota K. Impaired functional visual acuity of dry eye patients. Am J Ophthalmol. 2002; 133(2):181-186. [PubMed: 11812420]

41. Montés-Micó R, Cáliz A, Alió JL. Wavefront analysis of higher order aberrations in dry eye patients. J Refract Surg. 2004; 20(3):243-247. [PubMed: 15188901]

42. Lu L, Reinach PS, Kao WW. Corneal epithelial wound healing. Exp Biol Med (Maywood). 2001; 226(7):653-664. [PubMed: 11444101]

43. Belmonte C, Acosta MC, Gallar J. Neural basis of sensation in intact and injured corneas. Exp Eye Res. 2004; 78(3):513-525. [PubMed: 15106930] 


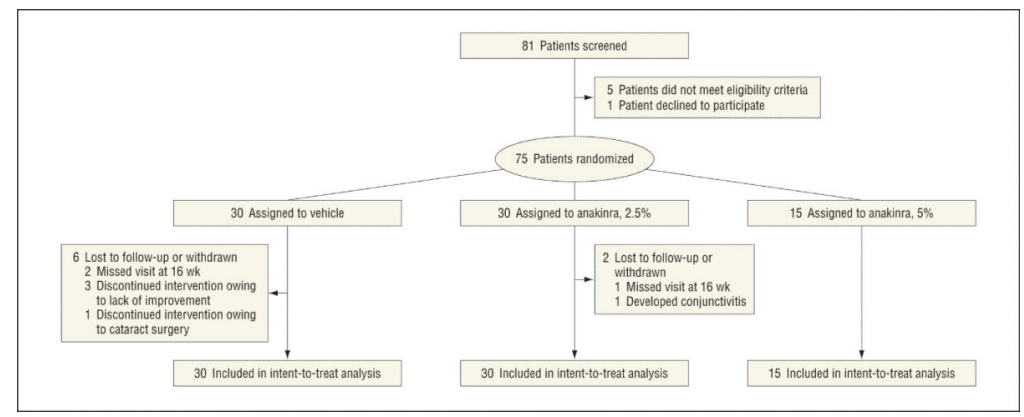

Figure 1.

Consolidated Standards of Reporting Trials flow diagram of the study. Eighty-one patients were screened, and 6 were excluded at this stage. Seventy-five patients were randomized to the 3 arms of the trial. Eight patients withdrew before the last study visit at week 16. 


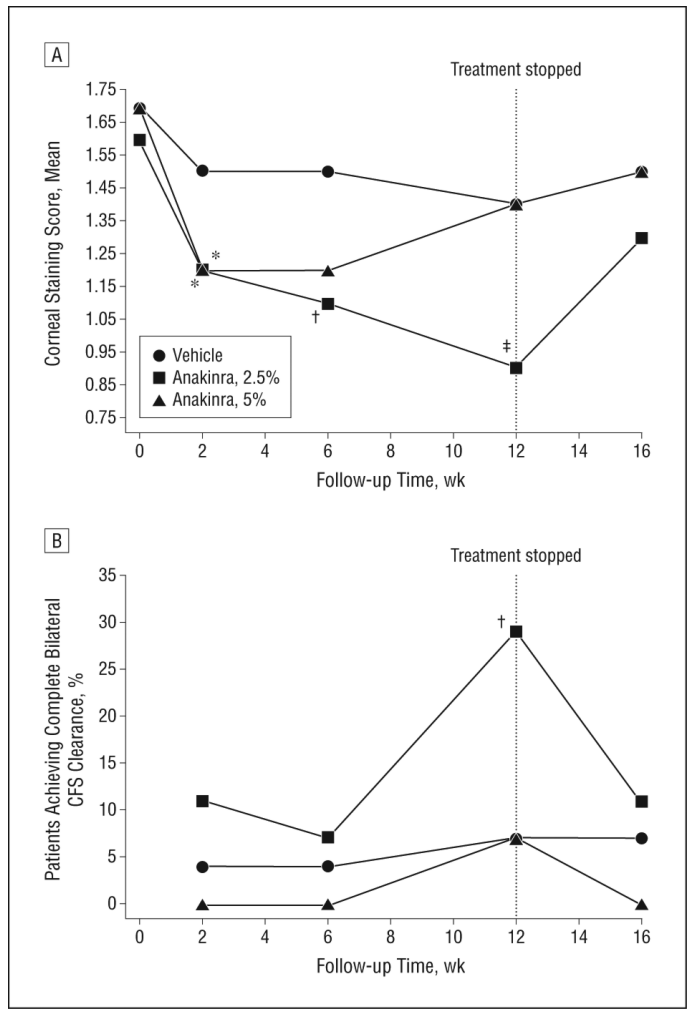

Figure 2.

Corneal fluorescein staining (CFS) (epitheliopathy) scores. A, The mean corneal staining score during 16 follow-up weeks. B, Proportions of patients in each treatment arm who recovered from epithelial fluorescein staining in both eyes (corneal clearance). The dashed vertical line at week 12 indicates the end of the treatment period. $* P<.01, \dagger P<.05$, and $\ddagger P$ $<.001$ for the comparison of each time point value with the baseline value. The mean changes in corneal staining between anakinra, $2.5 \%$, and anakinra, 5\%, compared with vehicle at week $6(P=.33$ and $P=.38$, respectively $)$ and week $12(P=.12$ and $P=.88$, respectively) did not reach statistical significance. At week 12, anakinra, $2.5 \%$, attained a statistically significant difference compared with vehicle in achieving complete bilateral CFS clearance $(P=.03$ and $P=.71$, respectively). 


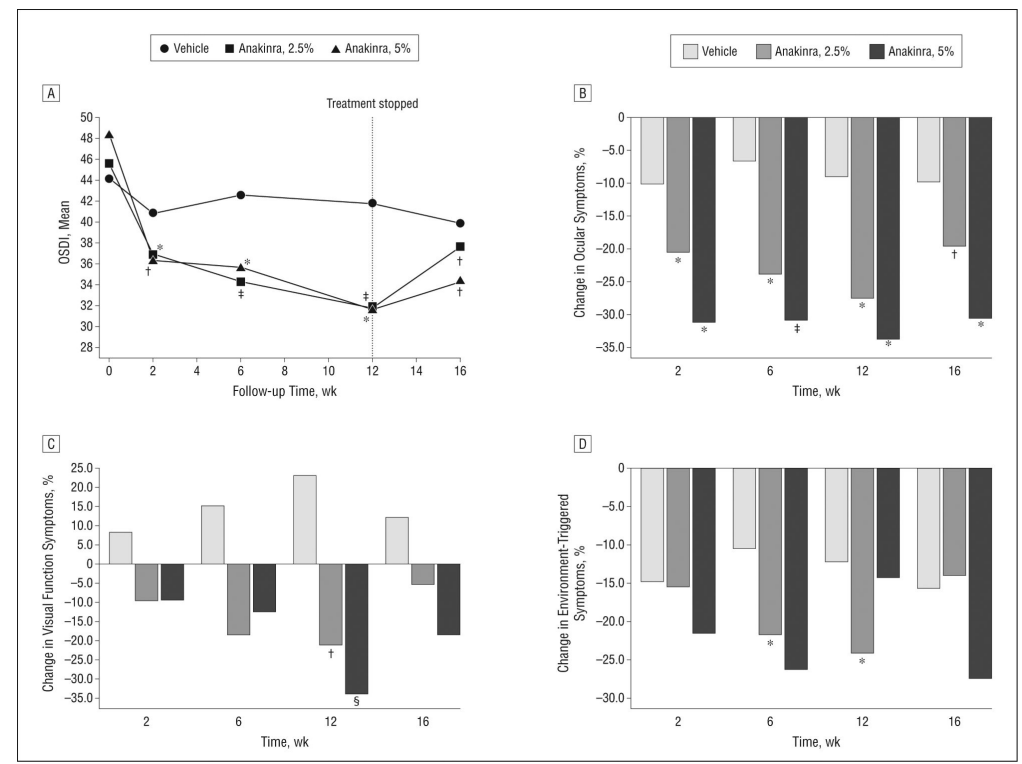

Figure 3.

Symptom scores. A, The mean Ocular Surface Disease Index (OSDI) during 16 follow-up weeks. The dashed vertical line at week 12 indicates the end of the treatment period. B, Percentage change in the Ocular Symptoms module of the OSDI questionnaire for each time point compared with baseline. C, Percentage change in the Visual Function Symptoms module of the OSDI questionnaire. D, Percentage change in the Environment-Triggered Symptoms module of the OSDI questionnaire. ${ }^{*} P<.01, \dagger P<.05, \ddagger P<.001$, and $\S P=.05$ for the comparison of each time point value with the baseline value. Both anakinra, 2.5\%, and anakinra, $5 \%$, led to a statistically significant decrease in dry eye symptom scores compared with vehicle at week $6(P=.02$ and $P=.02$, respectively) and week $12(P=.02$ and $P=.01$, respectively). 


\section{Table 1}

\section{Patient Baseline Characteristics}

\begin{tabular}{|c|c|c|c|}
\hline Characteristic & Vehicle $(\mathbf{n}=\mathbf{3 0})$ & Anakinra, $2.5 \%(n=30)$ & Anakinra, $5 \%(\mathrm{n}=15)$ \\
\hline Age, mean (SD), y & $53(12)$ & $49(14)$ & $58(16)$ \\
\hline \multicolumn{4}{|l|}{ Sex, No. $(\%)$} \\
\hline Male & $14(47)$ & $12(40)$ & $5(33)$ \\
\hline Female & $16(53)$ & $18(60)$ & $10(67)$ \\
\hline CFS score of both eyes, mean (SD) ${ }^{a}$ & $1.7(1.2)$ & $1.6(0.9)$ & $1.7(0.9)$ \\
\hline Complete bilateral CFS clearance, No. $(\%)$ & $2(7)$ & $2(7)$ & 0 \\
\hline Dry eye-related symptoms, mean (SD) ${ }^{b}$ & $44.2(20.4)$ & $45.6(21.1)$ & $48.5(21.0)$ \\
\hline Tear film breakup time of both eyes, mean (SD), $\mathrm{s}$ & $3.8(2.1)$ & $3.8(2.8)$ & $3.5(2.2)$ \\
\hline $\begin{array}{l}\text { Meibomian gland secretion quality of both eyes, mean } \\
(\mathrm{SD})^{c}\end{array}$ & $1.9(0.4)$ & $1.8(0.5)$ & $1.9(0.5)$ \\
\hline $\begin{array}{l}\text { Schirmer tear secretion test score of both eyes, mean (SD), } \\
\text { mm during } 5 \text { min }\end{array}$ & $5.2(3.3)$ & $6.5(6.0)$ & $5.6(4.7)$ \\
\hline $\begin{array}{l}\text { Expressible meibomian glands, mean (SD), No. of glands } \\
\text { among } 10 \text { examined }\end{array}$ & $6.0(2.3)$ & $6.3(2.3)$ & $6.8(2.0)$ \\
\hline $\begin{array}{l}\text { Visual acuity of both eyes, mean (SD), logMAR U } \\
\text { [Snellen equivalent] }\end{array}$ & $0.071(0.091)[20 / 23.55]$ & $0.103(0.195)$ [20/25.35] & $0.103(0.128)[20 / 25.35]$ \\
\hline Intraocular pressure of both eyes, mean (SD), $\mathrm{mm} \mathrm{Hg}$ & $13.7(3.4)$ & $12.9(3.1)$ & $13.8(3.9)$ \\
\hline
\end{tabular}

Abbreviation: CFS, corneal fluorescein staining.

${ }^{a}$ Range of 0 to 5 (representing increasing disease severity) on the modified Oxford Grading Scale. ${ }^{23}$

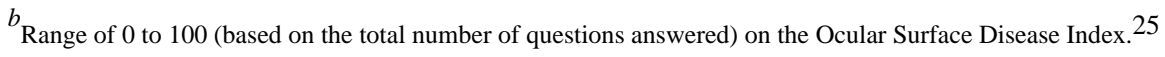

${ }^{c}$ Meibomian gland secretion quality has a range of 0 (normal) to 3 (abnormal). ${ }^{24}$ 


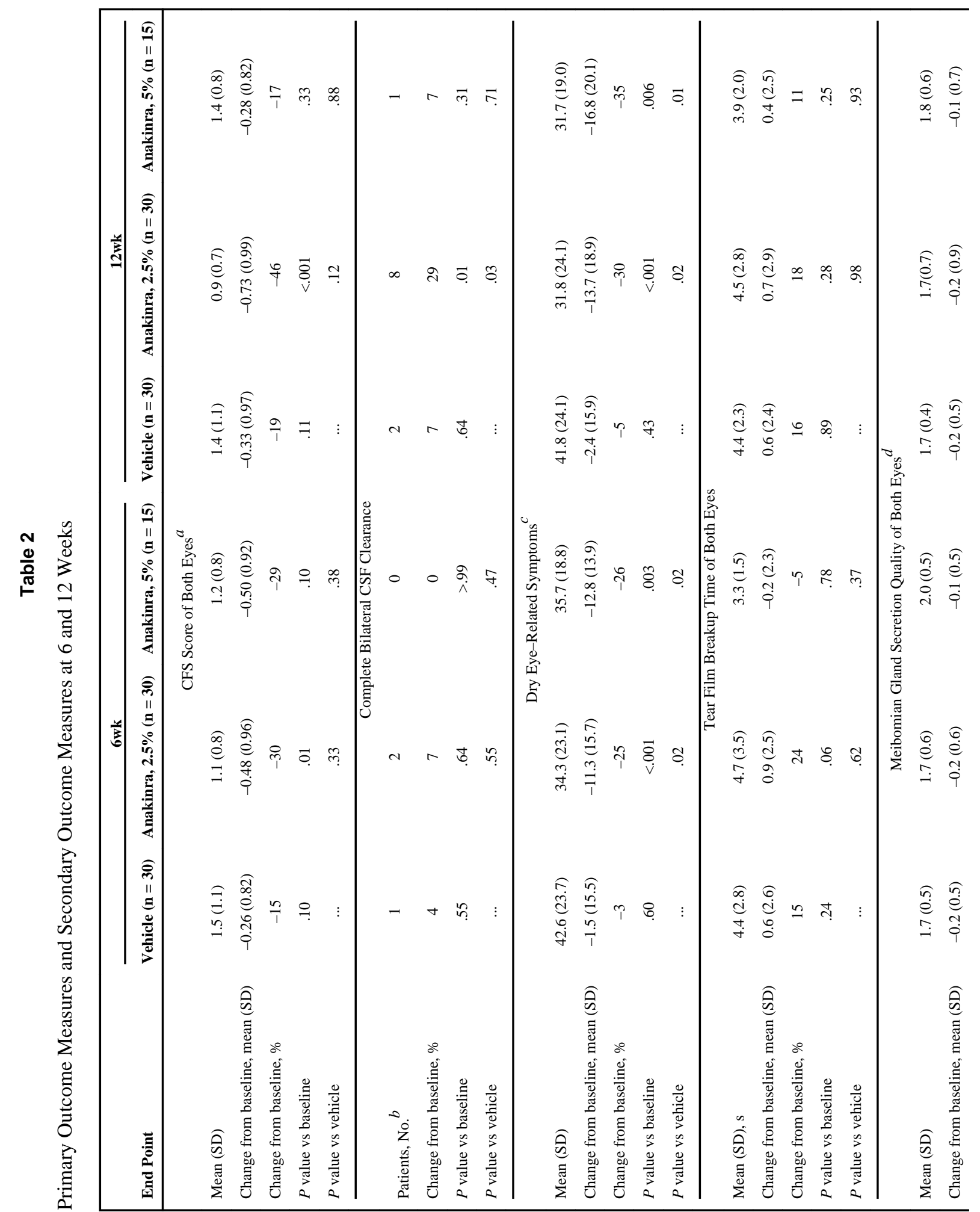









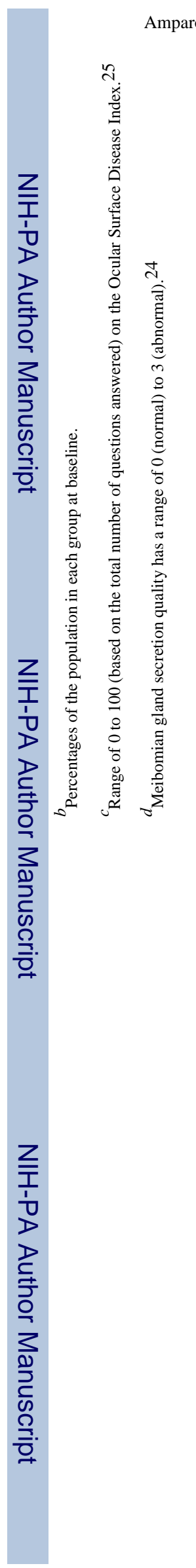

Page 18 